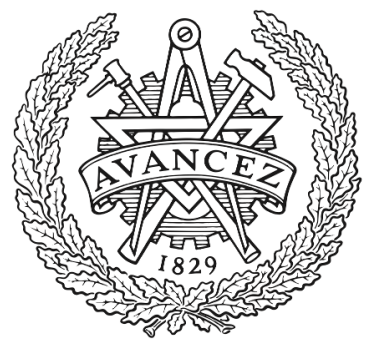

CHALMERS

UNIVERSITY OF TECHNOLOGY

\title{
Statistical analysis of antenna array systems with perturbations in phase, gain and element positions
}

Downloaded from: https://research.chalmers.se, 2023-04-26 14:20 UTC

Citation for the original published paper (version of record):

Moghaddam, M., Rezaei Aghdam, S., Eriksson, T. (2019). Statistical analysis of antenna array systems with perturbations in phase, gain and element positions. GlobalSIP 2019 - 7th IEEE Global Conference on Signal and Information Processing, Proceedings. http://dx.doi.org/10.1109/GlobalSIP45357.2019.8969083

N.B. When citing this work, cite the original published paper. 


\section{Statistical Analysis of Antenna Array Systems with Perturbations in Phase, Gain and Element Positions}

\author{
Mohammad Hossein Moghaddam \\ Department of Electrical Engineering \\ Chalmers University of Technology \\ Gothenburg, Sweden \\ mh.moghaddam@chalmers.se
}

\author{
Sina Rezaei Aghdam \\ Department of Electrical Engineering \\ Chalmers University of Technology \\ Gothenburg, Sweden \\ sinar@chalmers.se
}

\author{
Thomas Eriksson \\ Department of Electrical Engineering \\ Chalmers University of Technology \\ Gothenburg, Sweden \\ thomase@chalmers.se
}

\begin{abstract}
In this paper, we statistically analyze the effect of hardware impairments on power pattern of antenna array systems. We consider a linear array and formulate the stochastic beam pattern as a function of variations in phase, gain and element positions. By deriving a closed-form expression for the variance of the power pattern, we express how the performance of antenna array can be degraded in each angle, allowing for investigation of the role of each parameter in the final power pattern variations. The proposed closed-form expression serves as a tractable tool for analyzing the effect of perturbations in different settings.
\end{abstract}

Index Terms-Antenna array, phase errors, hardware impairments, array factor, power pattern variation.

\section{INTRODUCTION}

The performance of antenna array systems is unavoidably affected by different impairments including perturbations in phase, gain and array positions which are introduced due to non-ideal manufacturing process, aging and environmental conditions [1]. These perturbations make the beam pattern deviate from the nominal one which can considerably degrade the system performance and typically, several steps of calibration are required for compensating their effects.

To quantify how much degradation can be tolerated due to the effect of such perturbations, and then to design proper calibration algorithms, one first needs to know the characteristics of the power pattern variations. Since the perturbations are random variables, it is desirable to characterize the power pattern variations in terms of the first and second order statistics. Several works have been done in the literature about analytical study of the impact of random perturbations on the performance of antenna array systems [2]-[7]. In [2], the mean of power pattern is calculated in terms of the mean of random perturbations in phase (steering vector). The work in [3] models the sidelobe level of an antenna array using Rician distribution and presents an approximation for the variance of beam pattern as a function of the variance of perturbations in phase and gain (complex gain vector). In [4], [5], the authors model the combined effect of different perturbations using a single multidimensional random variable and calculate the

This work is funded by European union horizon 2020 research and innovation program under the MARIE SKODOWSKA-CURIE agreement No 721732 for Silika project, http://silika-project.eu. variance of beam pattern with the aid of a-priori information on the statistics of perturbations. In [6], a closed-form function for the mean of power pattern is derived in terms of variances of phase, gain, and element position perturbations. In [7], the beam pattern in presence of gain and phase perturbations is assumed to be a circular-symmetric complex Gaussian distributed function and the mean and variance of the beam pattern are calculated based on that approximate assumption.

There are also a few other works in the literature which analyze the perturbations in antenna arrays based on numerical methods and Monte-Carlo simulations [8]-[12]. In [8], the authors consider random gain and phase perturbations, and use Monte-Carlo simulations to numerically find the variations of the beam pattern. Similarly, in [9], Monte-Carlo simulations are used to obtain the mean of power pattern, the variance of beam pattern, and the bias of beam pattern for random perturbations in complex gain, element positions and orientations. In [10], [11], authors employ the interval arithmetic (IA) to compute power pattern bounds for antenna array perturbations in complex gain but with a reduced computational effort compared to Monte-Carlo methods. In [12], the authors propose a method to calculate the variations of power pattern by approximating the antenna power values using the first order Taylor expansion with respect to the uncertain amplitude in order to further reduce the computational complexity of the IA methods.

Although numerical calculations of the variations of power pattern by using Monte-Carlo simulations are effective for finding the bounds of variations [8]-[12], they are not tractable for analyzing the effects of all possible error combinations with all their different settings. It is more desirable to use analytical methods which are tractable for any combination of perturbations, allowing for investigation of the role of each parameter in the final power pattern variations. The previous works on analytical analysis [2]-[7], have not presented any closed-form expression for the variations of the power pattern. In this paper, we will follow the previous works done in [6] for calculation of mean of power pattern, and calculate the variance of power pattern for perturbations in phase, gain and element positions. We present a closed-form expression for the variance of power pattern in terms of variances of those three 
perturbation parameters, and express analytical bounds for the power pattern.

The paper is organized as follows. In Section II, we make a brief review on antenna array systems and present the formulations for statistical definition of perturbations. In Section III, we explain the analytical derivations for the variance of power pattern with all its details. In Section IV, we validate the analytical study with simulations, and finally, the paper is concluded in Section V.

\section{SySTEM MODEL}

\section{A. Antenna Array System Review}

Consider a linear array of $N$ elements on z-axis with positions $\mathbf{p}_{0}, \mathbf{p}_{1}, \ldots, \mathbf{p}_{N-1}$ where $\mathbf{p}_{n}=\left[\begin{array}{lll}0 & 0 & p_{n z}\end{array}\right]^{T}$. The array manifold vector is defined as [6]

$$
\mathbf{v}(\mathbf{k})=\left[\begin{array}{llll}
e^{-j \mathbf{k} \mathbf{p}_{0}} & e^{-j \mathbf{k} \mathbf{p}_{1}} & \ldots & e^{-j \mathbf{k} \mathbf{p}_{N-1}}
\end{array}\right]^{T},
$$

where $\mathbf{k}$ is the wavenumber and is used to express the direction of any array with any coordinates which is defined as

$$
\mathbf{k}=\frac{2 \pi}{\lambda}[\sin (\theta) \cos (\psi) \quad \sin (\theta) \sin (\psi) \quad \cos (\theta)],
$$

where $\lambda$ is the wavelength, $\theta$ is the angle towards the $z$-axis and $\psi$ is the angle from the $x$-axis. Now, if we consider a weight filter for each element, the complex gain vector is defined as

$$
\mathbf{w}=\left[\begin{array}{llll}
w_{0} & w_{1} & \ldots & w_{N-1}
\end{array}\right]^{T},
$$

where,

$$
w_{i}=g_{i} e^{j \phi_{i}},
$$

is the complex gain of each element in the phase shifter network (PSN) and arises after vector modulator (VM) which includes a phase shifter (PS) and a variable gain amplifier (VGA). In case of uniform linear array, all element gains are equal to $1 / N$. The complex gain vector is the multiplication of real gains $g_{i}$ with steering vector elements $e^{j \phi_{i}}$. These two components have different effects on the beam pattern. The steering vector is used for changing the direction of the main beam, and subsequently will change the direction of sidelobes. The vector of real gains on the other hand, is usually used for tapering and reducing the side-lobe levels, which also has some effects on the main lobe and can change the shape of the beam pattern. Beam pattern is defined as [6]

$$
B(\theta, \psi)=B(\mathbf{k})=\mathbf{w}^{H} \mathbf{v}(\mathbf{k})=\sum_{i=0}^{N-1} g_{i} e^{j\left(\phi_{i}\right)} e^{-j \mathbf{k p}_{i}} .
$$

\section{B. Statistical Modeling of Perturbations}

If we consider the effect of perturbations in gain and phase of each element in the complex gain vector (2), then for each element we can write

$$
w_{i}=g_{i}\left(1+\Delta g_{i}\right) e^{j\left(\phi_{i}+\Delta \phi_{i}\right)},
$$

where $\Delta g_{i}$ and $\Delta \phi_{i}$ are random variables with variances $\sigma_{g}^{2}$ and $\sigma_{\phi}^{2}$.
We can define the perturbations in the position of array elements as

$$
\mathbf{p}_{i}=\mathbf{p}_{i}^{c}+\left[\begin{array}{lll}
0 & 0 & \Delta p_{i}
\end{array}\right]^{T}
$$

where $\Delta p_{i}$ is a random parameter with the variance of $\sigma_{\lambda}^{2}$ in $z-$ axis, and $\mathbf{p}_{i}^{c}$ is the vector of nominal element position in $3 \mathrm{D}$ space. Therefore, the stochastic beam pattern function can be written as

$$
B(\mathbf{k})=\sum_{i=0}^{N-1} g_{i}\left(1+\Delta g_{i}\right) e^{\left(j\left(\phi_{i}+\Delta \phi_{i}\right)-j \mathbf{k p}_{i}\right)} .
$$

Now, if we consider $\Delta p_{i}, \Delta g_{i}$ and $\Delta \phi_{i}$ for $i=0,1, \ldots, N-1$ as statistically independent, zero-mean, and Gaussian random variables [6], then the beam pattern in (8) is actually a random function of $\sigma_{g}^{2}, \sigma_{\phi}^{2}$ and $\sigma_{\lambda}^{2}$ as the variances of perturbations in gain, phase, and element positions. The expectation of magnitude square of beam pattern (power pattern) can be obtained as [6]

$$
\begin{aligned}
& \mathbb{E}\left[|B(\mathbf{k})|^{2}\right] \\
& =\left|B^{c}(\mathbf{k})\right|^{2} e^{-\left(\sigma_{\phi}^{2}+\sigma_{\lambda}^{2}\right)}+\left(\left(1+\sigma_{g}^{2}\right)-e^{-\left(\sigma_{\phi}^{2}+\sigma_{\lambda}^{2}\right)}\right) \sum_{i=0}^{N-1} g_{i}^{2},
\end{aligned}
$$

where $\left|B^{c}(\mathbf{k})\right|$ is the nominal beam pattern without any perturbation.

\section{Statistical Analysis of POWER PATtern VARIATIONS}

Eq. (9) gives us the average power, but we need to know the variance of the power pattern function to characterize its probable variations in the presence of perturbations. To mathematically formulate the variations, we need to calculate the variance of magnitude square of the beam pattern in (8). Namely, we need to find

$$
\Lambda=\mathbb{E}\left[|B(\mathbf{k})|^{4}\right]-\left(\mathbb{E}\left[|B(\mathbf{k})|^{2}\right]\right)^{2} .
$$

For the second term, we can directly use (9), and for the first term, according to the expression of expected value of a random variable to the power of four, we have

$$
\begin{gathered}
\mathbb{E}\left[|B(\mathbf{k})|^{4}\right]=\mathbb{E}\left[B(\mathbf{k})^{H} B(\mathbf{k}) B(\mathbf{k})^{H} B(\mathbf{k})\right] \\
=\sum_{i=0}^{N-1} \sum_{l=0}^{N-1} \sum_{m=0}^{N-1} \sum_{q=0}^{N-1} \mathbb{E}\left[g_{i}\left(1+\Delta g_{i}\right) g_{l}\left(1+\Delta g_{l}\right) g_{m}\left(1+\Delta g_{m}\right) g_{q}\right. \\
\left(1+\Delta g_{q}\right) e^{j\left(\phi_{i}+\Delta \phi_{i}-\phi_{l}-\Delta \phi_{l}+\phi_{m}+\Delta \phi_{m}-\phi_{q}-\Delta \phi_{q}\right)} \\
e^{-j \mathbf{k}\left(\mathbf{p}_{i}-\mathbf{p}_{l}+\mathbf{p}_{m}-\mathbf{p}_{q}\right]}
\end{gathered}
$$


Now, if we consider all random variables as statistically independent, zero-mean, and Gaussian, then by using method of moments, for the first part, we can write

$$
\begin{aligned}
& \mathbb{E}\left[\left(1+\Delta g_{i}\right)\left(1+\Delta g_{l}\right)\left(1+\Delta g_{m}\right)\left(1+\Delta g_{q}\right)\right] \\
& =\mathbb{E}\left[1+\Delta g_{m} \Delta g_{q}+\Delta g_{i} \Delta g_{m}+\Delta g_{i} \Delta g_{q}+\Delta g_{l} \Delta g_{m}\right. \\
& +\Delta g_{l} \Delta g_{q}+\Delta g_{i} \Delta g_{l}+\Delta g_{i} \Delta g_{m} \Delta g_{q}+\Delta g_{l} \Delta g_{m} \Delta g_{q} \\
& \left.+\Delta g_{i} \Delta g_{l} \Delta g_{m}+\Delta g_{i} \Delta g_{l} \Delta g_{q}+\Delta g_{i} \Delta g_{l} \Delta g_{m} \Delta g_{q}\right]
\end{aligned}
$$

where by any coordination of indices, the expectation of product of three random variables are zero and for the last term, by using the expression for the expected value of multiplication of four jointly Gaussian random variables [13], we can write

$$
\begin{aligned}
& \mathbb{E}\left[\Delta g_{i} \Delta g_{l} \Delta g_{m} \Delta g_{q}\right] \\
& =\mathbb{E}\left[\Delta g_{i} \Delta g_{l}\right] \mathbb{E}\left[\Delta g_{m} \Delta g_{q}\right]+\mathbb{E}\left[\Delta g_{i} \Delta g_{m}\right] \mathbb{E}\left[\Delta g_{l} \Delta g_{q}\right] \\
& +\mathbb{E}\left[\Delta g_{i} \Delta g_{q}\right] \mathbb{E}\left[\Delta g_{l} \Delta g_{m}\right]=\left(\delta_{i l} \delta_{m q}+\delta_{i m} \delta_{l q}+\delta_{i q} \delta_{l m}\right) \sigma_{g}^{4},
\end{aligned}
$$

which is derived using characteristic function of zero-mean jointly normal random variables, and $\delta_{i l}=1$ when $i=l$, and otherwise is zero. Now, we can rewrite equation (13) as

$$
\begin{aligned}
& \mathbb{E}\left[\left(1+\Delta g_{i}\right)\left(1+\Delta g_{l}\right)\left(1+\Delta g_{m}\right)\left(1+\Delta g_{q}\right)\right] \\
& =1+\left(\delta_{m q}+\delta_{i m}+\delta_{i q}+\delta_{l m}+\delta_{l q}+\delta_{i l}\right) \sigma_{g}^{2} \\
& +\left(\delta_{i l} \delta_{m q}+\delta_{i m} \delta_{l q}+\delta_{i q} \delta_{l m}\right) \sigma_{g}^{4} .
\end{aligned}
$$

By using (23) in the Appendix, the second term in (12) can be written as

$$
\mathbb{E}\left[e^{j\left(\Delta \phi_{i}-\Delta \phi_{l}+\Delta \phi_{m}-\Delta \phi_{q}\right)}\right]=e^{\mathbf{a}^{T} \mathbf{m}+0.5 \mathbf{a}^{T} \mathbf{\Sigma} \mathbf{a}},
$$

where $\mathbf{m}$ is the zero vector, since we have considered zeromean random variables, and the covariance matrix $\boldsymbol{\Sigma}$ and the vector a are defined as

$$
\begin{aligned}
& \boldsymbol{\Sigma}=\left[\begin{array}{cccc}
\sigma_{i i}^{2} & \sigma_{i l}^{2} & \sigma_{i m}^{2} & \sigma_{i q}^{2} \\
\sigma_{l i}^{2} & \sigma_{l l}^{2} & \sigma_{l m}^{2} & \sigma_{l q}^{2} \\
\sigma_{m i}^{2} & \sigma_{m l}^{2} & \sigma_{m m}^{2} & \sigma_{m q}^{2} \\
\sigma_{q i}^{2} & \sigma_{q l}^{2} & \sigma_{q m}^{2} & \sigma_{q q}^{2}
\end{array}\right], \\
& \mathbf{a}=\left[\begin{array}{llll}
j & -j & j & -j
\end{array}\right]^{T},
\end{aligned}
$$

respectively. We can rewrite (15) as

$$
\begin{aligned}
& \mathbb{E}\left[e^{j\left(\Delta \phi_{i}-\Delta \phi_{l}+\Delta \phi_{m}-\Delta \phi_{q}\right)}\right] \\
& =e^{0.5\left[-\left(\sigma_{i i}^{2}+\sigma_{l l}^{2}+\sigma_{m m}^{2}+\sigma_{q q}^{2}\right)+2\left(\sigma_{i l}^{2}-\sigma_{i m}^{2}+\sigma_{i q}^{2}+\sigma_{l m}^{2}-\sigma_{l q}^{2}+\sigma_{m q}^{2}\right)\right]} \\
& =e^{\sigma_{\phi}^{2}\left(-2+\left(\delta_{m q}-\delta_{i m}+\delta_{i q}+\delta_{l m}-\delta_{l q}+\delta_{i l}\right)\right)}
\end{aligned}
$$

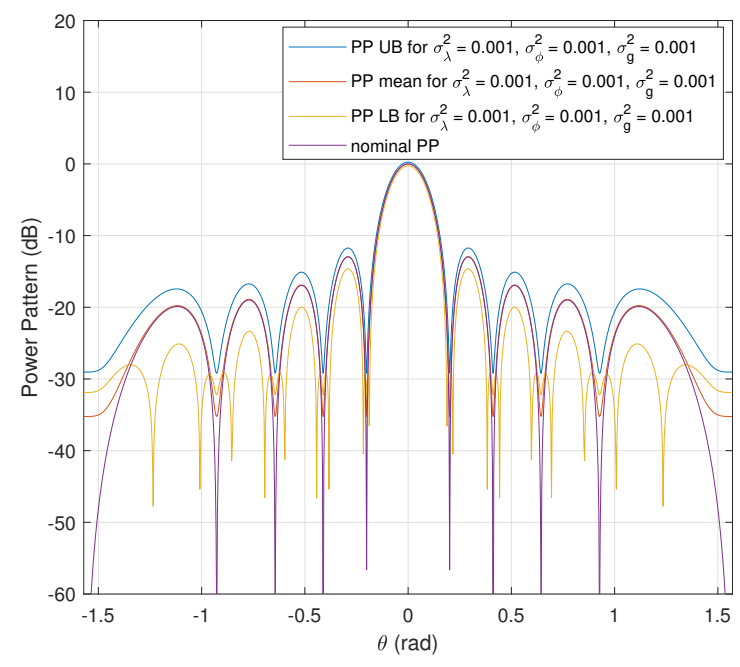

(a)

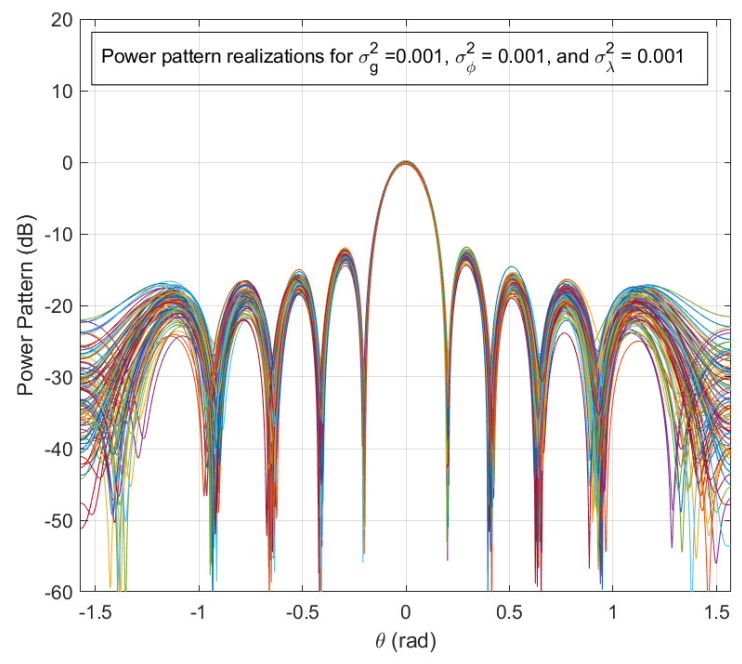

(b)

Fig. 1: Power pattern analysis for a) analytical derivations based on (9) and (20), and for b) Monte-Carlo realizations.

Finally, for the last multiplicative term in (12), by using (23) in the Appendix, we can write

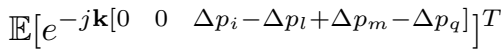

$$
\begin{aligned}
& =e^{\sigma_{\lambda}^{2}\left(-2+\left(\delta_{m q}-\delta_{i m}+\delta_{i q}+\delta_{l m}-\delta_{l q}+\delta_{i l}\right)\right)} .
\end{aligned}
$$

Thus, according to equation (12) and by using the results in equations (14), (18), and (19), we can express the variance of power pattern as given in (20) on top of the next page. This closed-form expression can give us very useful insights through the variations of the power pattern in presence of perturbations which is explained in the next section.

\section{Numerical Results}

For numerical analysis, we have considered an array of $N=10$ antenna elements symmetrically positioned on the zaxis with steering angle of $\theta=0$ in the broadside. The curves 


$$
\begin{array}{r}
\Lambda=\sum_{i=0}^{N-1} \sum_{l=0}^{N-1} \sum_{m=0}^{N-1} \sum_{q=0}^{N-1}\left[( g _ { i } g _ { l } g _ { m } g _ { q } e ^ { j ( \phi _ { i } - \phi _ { l } + \phi _ { m } - \phi _ { q } ) } e ^ { - j \mathbf { k } ( \mathbf { p } _ { i } ^ { c } - \mathbf { p } _ { l } ^ { c } + \mathbf { p } _ { m } ^ { c } - \mathbf { p } _ { q } ^ { c } ) } ) \left(1+\left(\delta_{m q}+\delta_{i m}+\delta_{i q}+\delta_{l m}+\delta_{l q}+\delta_{i l}\right) \sigma_{g}^{2}\right.\right. \\
\left.\left.+\left(\delta_{i l} \delta_{m q}+\delta_{i m} \delta_{l q}+\delta_{i q} \delta_{l m}\right) \sigma_{g}^{4}\right)\left(e^{\sigma_{\phi}^{2}\left(-2+\left(\delta_{m q}-\delta_{i m}+\delta_{i q}+\delta_{l m}-\delta_{l q}+\delta_{i l}\right)\right)}\right)\left(e^{\sigma_{\lambda}^{2}\left(-2+\left(\delta_{m q}-\delta_{i m}+\delta_{i q}+\delta_{l m}-\delta_{l q}+\delta_{i l}\right)\right)}\right)\right] \\
-\left(\left|B^{c}(\mathbf{k})\right|^{2} e^{-\left(\sigma_{\phi}^{2}+\sigma_{\lambda}^{2}\right)}+\left[\left(1+\sigma_{g}^{2}\right)-e^{-\left(\sigma_{\phi}^{2}+\sigma_{\lambda}^{2}\right)}\right] \sum_{i=0}^{N-1} g_{i}^{2}\right)^{2}
\end{array}
$$
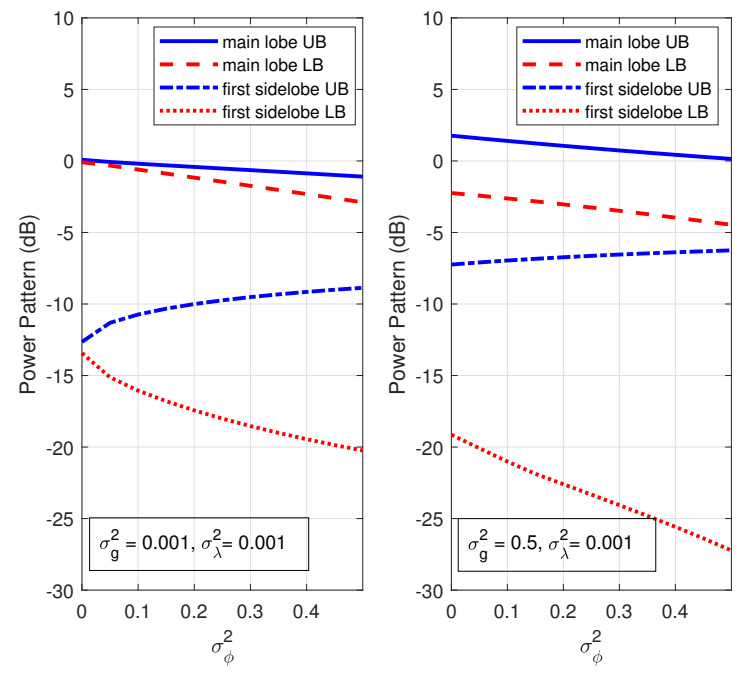

(a)
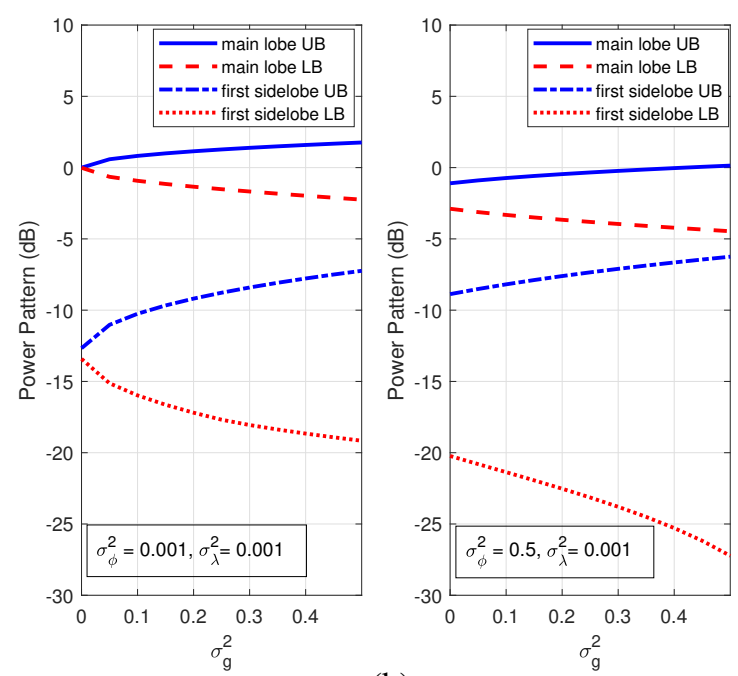

(b)

Fig. 2: Power pattern for the main lobe level and first sidelobe level with $\sigma_{\lambda}^{2}=0.001$ in a) span of $\sigma_{\phi}^{2}$, and b) span of $\sigma_{g}^{2}$.

for nominal power pattern (nominal PP), the mean of power pattern (PP mean) based on (9), three standard deviations higher than the mean of the power pattern (PP UB), and three standard deviations lower than the mean of the power pattern (PP LB) based on (20) are depicted in Fig. 1-a. In Fig. 2-b, the power pattern is depicted for 100 realizations based on (8).
The standard deviation based on (20) is calculated as 0.020 for the main-lobe level at $\theta=0$, which is closely matched to 0.0188 as the standard deviation calculated numerically from 100 realizations for the same angle $(\theta=0)$. These results reveal the accuracy of (20).

In Fig. 2, the upper bounds and lower bounds of power pattern are depicted for the main-lobe and the first side-lobe levels in different settings of perturbations. According to the figures, we can investigate in each combination of perturbations, which one is the most affecting one that deteriorates the performance. The proposed formulation also facilitates error tolerance studies. For example, according to Fig. 2-b, we can say that for the tolerance level of up to one $\mathrm{dB}$ in the main beam ( $1 \mathrm{~dB}$ loss), the variance of perturbations in gain should be less than $0.1\left(\sigma_{g}^{2}<0.1\right)$ with $\sigma_{\lambda}^{2}=0.001$ and $\sigma_{\phi}^{2}=0.001$.

\section{CONClusion}

We have formulated a closed-form expression for variance of power pattern in antenna array systems. This closed-form function can predict the behavior of beam pattern for any combination of perturbations in gain, phase and antenna positions, and is a reliable metric for antenna array performance analysis, distinguishing the dominant perturbations, and it can be used for impairment calibrations.

\section{APPENDIX}

Considering a Normal random variable as $Z \sim \mathcal{N}\left(\mu, \sigma^{2}\right)$, then the $n$th moment of $Z$ will be [13]

$$
M_{Z}(n)=e^{\left(\mu n+\sigma^{2} n^{2} / 2\right)},
$$

and then we have

$$
\mathbb{E}\left[e^{Z}\right]=M_{Z}(1)=e^{\left(\mu+\sigma^{2} / 2\right)} .
$$

In general, with $\mathbf{a}=\left[\begin{array}{lll}a_{1} & \ldots & a_{K}\end{array}\right]^{T}$ and $\mathbf{z}=\left[\begin{array}{lll}z_{1} & \ldots & z_{K}\end{array}\right]^{T}$ and by using general moment function, we can write the expected value of product of normal random variables as

$$
\mathbb{E}\left[\prod_{i=1}^{K} e^{a_{i} z_{i}}\right]=\mathbb{E}\left[e^{\mathbf{a}^{T} \mathbf{z}}\right]=e^{\mathbf{a}^{T} \mathbf{m}+0.5 \mathbf{a}^{T} \Sigma \mathbf{a}},
$$

where $\mathbf{m}=\left[\begin{array}{lll}\mu_{1} & \ldots & \mu_{K}\end{array}\right]^{T}$ is the vector of mean values, and $\boldsymbol{\Sigma}$ is the covariance matrix. 


\section{REFERENCES}

[1] B. Allen and M. Ghavami, Adaptive Array Systems: Fundamentals and Applications, Wiley, 2006.

[2] K Carver, W. Cooper, and W Stutzman, "Beam-pointing errors of planarphased arrays," IEEE Trans. Antennas Propag., vol. 21, no. 2, pp. 199202, 1973.

[3] J. K. Hsiao, "Design of error tolerance of a phased array," Electronics Letters, vol. 21, no. 19, pp. 834-836, 1985.

[4] M. Viberg and A. L. Swindlehurst, "Analysis of the combined effects of finite samples and model errors on array processing performance," in 1993 IEEE Int. Conf. Acoust., Speech, Signal Process. (ICASSP), 1993, vol. 4, pp. 372-375.

[5] M. Viberg and A. L. Swindlehurst, "A bayesian approach to autocalibration for parametric array signal processing," IEEE Trans. Signal Process., vol. 42, no. 12, pp. 3495-3507, 1994.

[6] H. L. Van Trees, Optimum array processing: Part IV of detection, estimation, and modulation theory, John Wiley \& Sons, 2004.

[7] P. K. Tam, K.T. Wong, and Y. Song, "An hybrid Cramer-Rao bound in closed form for direction-of-arrival estimation by an acoustic vector sensor with gain-phase uncertainties," IEEE Trans. Signal Process., vol. 62, no. 10, pp. 2504-2516, 2014.

[8] J. Lee, Y. Lee, and H. Kim, "Decision of error tolerance in array element by the Monte Carlo method," IEEE Trans. Antennas Propag., vol. 53, no. 4, pp. 1325-1331, 2005.

[9] A. J Poulsen, R. R. Nadakuditi, and A. B. Baggeroer, "Robust adaptive vector sensor processing in the presence of mismatch and finite sample support," in 2008 5th IEEE Sensor Array and Multichannel Signal Processing Workshop. IEEE, 2008, pp. 473-477.

[10] A. Massa, P. Rocca, E. Giaccari, and A. Farina, "Tolerance analysis of antenna arrays through interval analysis," in 2014 IEEE Radar Conf., 2014, pp. 0896-0899.

[11] L. Poli, P. Rocca, N. Anselmi, and A. Massa, "Dealing with uncertainties on phase weighting of linear antenna arrays by means of interval-based tolerance analysis," IEEE Trans. Antennas Propag., vol. 63, no. 7, pp. $3229-3234,2015$

[12] N. Hu, B. Duan, W. Xu, and J. Zhou, "A new interval pattern analysis method of array antennas based on Taylor expansion," IEEE Trans. Antennas Propag., vol. 65, no. 11, pp. 6151-6156, 2017.

[13] A. Papoulis and S. U. Pillai, Probability, random variables, and stochastic processes, p. 258, Tata McGraw-Hill Education, 2002. 